\title{
PROspective Multicenter Imaging Study for Evaluation of chest pain: Rationale and design of the PROMISE trial
}

\section{Citation}

Douglas, Pamela S., Udo Hoffmann, Kerry L. Lee, Daniel B. Mark, Hussein R. Al-Khalidi, Kevin Anstrom, Rowena J. Dolor, et al. 2014. “PROspective Multicenter Imaging Study for Evaluation of Chest Pain: Rationale and Design of the PROMISE Trial." American Heart Journal 167 (6) (June): 796-803.e1. doi:10.1016/j.ahj.2014.03.003.

\section{Published Version}

doi:10.1016/j.ahj.2014.03.003

\section{Permanent link}

http://nrs.harvard.edu/urn-3:HUL.InstRepos:29048901

\section{Terms of Use}

This article was downloaded from Harvard University's DASH repository, and is made available under the terms and conditions applicable to Other Posted Material, as set forth at http:// nrs.harvard.edu/urn-3:HUL.InstRepos:dash.current.terms-of-use\#LAA

\section{Share Your Story}

The Harvard community has made this article openly available.

Please share how this access benefits you. Submit a story.

Accessibility 


\title{
PROspective Multicenter Imaging Study for Evaluation of Chest Pain: Rationale and Design of the PROMISE Trial
}

\author{
Pamela S. Douglas, MD¹, Udo Hoffmann, MD, MPH${ }^{2}$, Kerry L. Lee, $\mathrm{PhD}^{1}$, Daniel B. Mark, \\ MD, MPH ${ }^{1}$, Hussein R. Al-Khalidi, PhD ${ }^{1}$, Kevin Anstrom, PhD $^{1}$, Rowena J. Dolor, MD, MHS ${ }^{1}$, \\ Andrzej Kosinski, PhD ${ }^{1}$, Mitchell W. Krucoff, MD ${ }^{1}$, Daniel W. Mudrick, MD, MPH ${ }^{1,3}$, Manesh \\ R. Patel, MD ${ }^{1}$, Michael H. Picard, MD², James E. Udelson, MD ${ }^{4}$, Eric J. Velazquez, MD ${ }^{1}$, and \\ Lawton Cooper, MD ${ }^{5}$ on behalf of the PROMISE investigators \\ ${ }^{1}$ Duke Clinical Research Institute, Duke University School of Medicine \\ ${ }^{2}$ Massachusetts General Hospital, Harvard Medical School \\ ${ }^{3}$ McConnell Heart Health Center, Columbus, $\mathrm{OH}$ \\ ${ }^{4}$ Tufts Medical Center and Tufts University School of Medicine \\ ${ }^{5}$ National Heart, Lung, and Blood Institute
}

\section{Abstract}

Background-Suspected coronary artery disease (CAD) is one of the most common, potentially life threatening diagnostic problems clinicians encounter. However, no large outcome-based randomized trials have been performed to guide the selection of diagnostic strategies for these patients.

Methods-The PROMISE study is a prospective, randomized trial comparing the effectiveness of two initial diagnostic strategies in patients with symptoms suspicious for CAD. Patients are randomized to either: 1) functional testing (exercise electrocardiogram, stress nuclear imaging, or stress echocardiogram); or 2) anatomic testing with $>=64$ slice multidetector coronary computed tomographic angiography. Tests are interpreted locally in real time by subspecialty certified physicians and all subsequent care decisions are made by the clinical care team. Sites are provided results of central core lab quality and completeness assessment. All subjects are followed for $\geq 1$ year. The primary end-point is the time to occurrence of the composite of death, myocardial infarction, major procedural complications (stroke, major bleeding, anaphylaxis and renal failure) or hospitalization for unstable angina.

Results-Over 10,000 symptomatic subjects were randomized in 3.2 years at 193 US and Canadian cardiology, radiology, primary care, urgent care and anesthesiology sites.

(C) 2014 Mosby, Inc. All rights reserved.

Corresponding author: Pamela S. Douglas, MD, 7022 North Pavilion DUMC, PO Box 17969, Durham, NC 27715, phone 919.681.2690, fax 919.668.7059.

Publisher's Disclaimer: This is a PDF file of an unedited manuscript that has been accepted for publication. As a service to our customers we are providing this early version of the manuscript. The manuscript will undergo copyediting, typesetting, and review of the resulting proof before it is published in its final citable form. Please note that during the production process errors may be discovered which could affect the content, and all legal disclaimers that apply to the journal pertain. 
Conclusion-Multi-specialty community practice enrollment into a large pragmatic trial of diagnostic testing strategies is both feasible and efficient. PROMISE will compare the clinical effectiveness of an initial strategy of functional testing against an initial strategy of anatomic testing in symptomatic patients with suspected CAD. Quality of life, resource use, cost effectiveness and radiation exposure will be assessed.

Clinical trials.gov identifier NCT01174550

\section{Keywords}

Angina; Coronary computed tomography angiogram; Diagnostic strategy; Stress ECG; Stress echocardiography; Stress nuclear

Chest pain is a common reason for patients to seek medical attention, with $\sim 4$ million Americans receiving a new diagnosis of angina each year.(1),(2) American College of Cardiology (ACC)/American Heart Association (AHA) guidelines recognize the utility of many forms of noninvasive diagnostic testing, including exercise electrocardiogram (ExECG), stress echocardiogram (SE), stress nuclear imaging(MPI), and coronary computed tomographic angiography (CCTA), but do not provide strong recommendations as to which is preferred.(3) The most commonly performed functional tests rely upon detecting stressinduced myocardial ischemia(3), and are associated with high rates of false-positive and false-negative results in the detection of obstructive CAD. A recent meta-analysis of functional testing reported sensitivities of 0.84 (95\% confidence interval:0.79-0.90) for SE and 0.86 (CI:0.81-0.91) for MPI; and specificities and areas under the curve of 0.80 (CI: 0.73-0.87) and 0.90 for SE and 0.82 (CI:0.75-0.89) and 0.91 for MPI.(4) However, these tests have limited prognostic discrimination, and an inability to detect nonobstructive CAD, which may also affect prognosis.(5-7) In addition, current testing patterns include low risk populations in whom current forms of testing may not provide optimal test performance.(810) The consequences of these limitations is seen in the high annual costs of noninvasive testing(11) and infrequent referral rate (roughly 10\%) of those tested for additional investigations.(8) Despite this low referral rate, the likelihood of not finding obstructive CAD at diagnostic cardiac catheterization is quite high at over $60 \%,(10)$ leading to calls for noninvasive functional testing to better justify its current use as the optimal 'gatekeeper' for this invasive procedure.(12)

In contrast, CCTA permits direct visualization of the coronary arteries, allowing noninvasive detection of significant stenoses and exclusion of CAD with greater accuracy than functional tests. CCTA has been extensively validated against the gold standard of invasive coronary angiography.(13-15) A recent meta-analysis of 89 studies demonstrated a mean sensitivity of 0.97 (95\% CI, 0.96-0.98) and mean specificity of $0.87(0.85-0.90)$ for the detection of obstructive CAD.(16) CCTA also extends the spectrum of disease by detecting prognostically important nonobstructive lesions and coronary plaque,(17-19) but has been associated with greater downstream testing, interventions, and costs,(20) possibly mediated by its higher sensitivity for identifying obstructive $\mathrm{CAD}$ as compared to functional testing; lower specificity in patients with coronary artery calcification or impaired image quality; and by detecting anatomic CAD that may be unrelated to patient symptoms. Nevertheless, in a low prevalence population, the ability of CCTA to accurately and efficiently exclude 
obstructive CAD may provide important clinical and economic advantages. Alternatively, invasive studies have shown improved outcomes in patients undergoing revascularization guided by physiologic (i.e. functional) information as compared to lesion stenosis alone (i.e. anatomic information).(21) Thus, the appropriate role of CCTA in stable chest pain patients remains undetermined.(22)

\section{Objectives}

The primary objective of the PROMISE trial is to compare the clinical outcomes of an initial diagnostic strategy of anatomic testing with CCTA to a usual care strategy of functional noninvasive testing in subjects with stable symptoms suspicious for CAD. Secondary objectives include comparison of the rate of finding no obstructive CAD on catheterization, health care costs, resource utilization, and patient reported quality of life (QOL). The principal safety objective is to compare radiation exposure by diagnostic testing assignment. Additional pre-specified exploratory analyses include assessment of testing quality and performance, including diagnostic accuracy, prognostic performance and concordance between the site and core lab interpretations. Finally, the PROMISE trial will define the feasibility and desirability of evaluating diagnostic testing strategies by performing a large, comparative effectiveness trial with clinical outcomes as the primary endpoint, an approach which has been championed but remains unproven. $(23,24)$

\section{Primary Hypothesis}

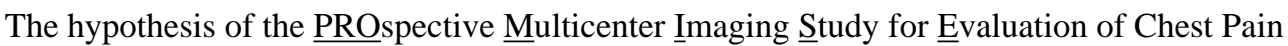
(PROMISE) is that an initial anatomic testing strategy utilizing coronary computed tomographic angiography (CCTA) technology will reduce the composite primary endpoint (all-cause death, myocardial infarction (MI), major peri-procedural complications, or hospitalization for unstable angina) by $20 \%$ when compared with an initial functional testing strategy over an average follow-up of 2.5 years. In the absence of prior controlled trial experience, this hypothesis is supported by the following considerations: 1) CCTA is more sensitive in detecting obstructive CAD leading to fewer false negative findings and untreated CAD; 2) CCTA is more specific in excluding obstructive disease leading to fewer false positive findings and unnecessary invasive therapies; 3) CCTA has greater prognostic accuracy; 4) CCTA is able to detect nonobstructive disease and therefore can optimize 'primary' prevention; and 5) physician confidence in the exclusion of CAD by CCTA is greater such that unnecessary catheterizations or hospital admissions for suspected disease will be reduced.

\section{Methods}

\section{Funding}

This project was supported by R01HL098237, R01HL098236, R01HL98305 and R01HL098235 from the National Heart, Lung, and Blood Institute (NHLBI). The authors are solely responsible for the design and conduct of this study, all study analyses, the drafting and editing of the paper and its final contents. This paper does not necessarily represent the official views of NHLBI. 


\section{Overall Design}

PROMISE is a multicenter, randomized, pragmatic trial comparing two state-of-the-art diagnostic strategies for use in patients with symptoms possibly due to obstructive CAD. (Figure 1) To establish an evidence-based standard-of-care PROMISE is constructed as an imaging effectiveness trial, as both efficacy (i.e., optimal performance at expert sites) and diagnostic utility or accuracy approaches would fail to capture the actual clinical performance of diagnostic strategies in the community where the vast majority of these tests are performed. Key features of a pragmatic design(25) are incorporated, including 1) the clinically relevant alternatives of usual clinical testing and usual clinical care; 2) a diverse study population; 3) heterogeneous practice settings; and 4) use of a broad range of health outcomes rather than focusing solely on cost. As is the case with many pragmatic trials, there is no blinding; this would be logistically impossible given the study design.

\section{Study population}

Subjects considered for enrollment into PROMISE were outpatients without known CAD who were symptomatic, and whose physician had determined that the subject required nonurgent, noninvasive cardiovascular testing to further evaluate suspected CAD. This population was purposefully and carefully chosen to be directly reflective of the population in which elective noninvasive cardiac diagnostic testing and cardiac catheterizations are currently being used. $(8,10)$ Inclusion Criteria were designed to capture at risk individuals with stable chest pain or chest pain equivalent syndromes, who had no history of coronary artery disease or recent evaluations for CAD. (Table 1). Exclusion Criteria were designed to exclude any individual with unstable hemodynamics or requiring urgent evaluation for suspected acute coronary syndrome (ACS), with a history of coronary artery disease, recent evaluations for $\mathrm{CAD}$, or who would be unable to be safely randomized to either arm.

\section{Site selection, qualification and imaging quality control}

To optimize the generalizability of the study results and reflect current patterns of care, community-based practices were aggressively recruited for participation in the trial, including primary care, radiology, cardiology and hospital based practices. Before beginning enrollment, potential sites were certified in each testing modality based on reader qualification, lab accreditation and successful transfer of 2 complete data sets with adequate image quality and completeness for each modality. For both CCTA and functional test readers, ACC COCATS (Core Cardiology Training Symposium) Level 2 training (26) or equivalent was required (for CCTA: Society of Cardiovascular Computed Tomography Level 2 or the Certification Board of Computed Cardiovascular Tomography; for nuclear, Certification Board of Nuclear Cardiology or Board Certification in nuclear medicine or radiology). As an additional eligibility criterion, CCTA level 2 readers were required to participate in an online course including evaluation of 25 cases of CCTA cases with comparison to invasive coronary angiography.

\section{Randomization}

Eligible subjects providing written informed consent were randomly assigned in a ratio of 1:1 to either the anatomic or functional diagnostic testing arm of the trial. A computer- 
generated permuted block randomization scheme with stratification by clinical site was used. Before randomization, the functional test that would be used if the subject were randomized to this arm was used as another stratification factor in the randomization scheme to facilitate comparisons of anatomic vs. functional testing according to the type of functional test.

\section{Diagnostic Testing Intervention}

In the functional testing arm, the preselected functional test was performed as the initial test: (MPI, stress echo, or ExECG). In the anatomic testing arm, a contrast-enhanced CCTA was performed as the initial test. Sites used standard equipment for functional testing as defined in current practice guidelines $(27,28)$ and $\succeq 64$-slice multidetector CCTA.(3) While sample protocols were provided for all modalities, sites were allowed to use their own acquisition protocols as long as they fell within national standard-of-care guidelines.(27-30) All diagnostic tests were interpreted by qualified diagnosticians at the sites in real time according to current clinical guidelines to ensure timely availability of results for patient management.

During the study, technical quality was assessed on all studies and $10 \%$ of studies were over-read centrally. Radiation exposure was monitored throughout the trial with a prespecified radiation safety plan in place. Sites not meeting set standards for radiation exposure underwent protocol review and retraining. No site was recommended for discontinuation of enrollment based on excessive radiation exposure.

\section{Subsequent Care}

The results of all tests were provided to the care team in the usual manner for that testing laboratory and the local physician continued to direct care of the subject, making all subsequent clinical decisions (e.g., need for further evaluation or admission) based upon his or her cumulative clinical assessment of the subject, including noninvasive test findings. Optimal medical therapy was encouraged and patient and provider educational materials were provided, including test-information sheets specific to each modality outlining diagnostic and prognostic implications of various test results.

\section{Follow-up visits}

Subjects had either a telephone call or clinic visit at $60(+/-14)$ days by the enrolling site for outcome evaluation, collection of test results and images and recording of any test complications. After that, subjects were contacted centrally by trained interviewers at 6 months post-randomization and at 6-month intervals for subsequent follow-up assessments until death, withdrawal, or the end of the trial. All subjects are followed for a minimum of one year. Quality of Life assessments were performed in the first 6000 subjects at baseline, and 6, 12 and 24 months.

\section{Effectiveness and Safety Determinations}

Endpoints were chosen to reflect clinical outcomes of importance to patients rather than intermediate outcomes like test utility or costs, which may change substantially depending on reimbursement policies. An independent clinical events adjudication committee (CEC) 
reviewed all primary endpoint events and the secondary endpoints which included catheterization in a blinded fashion based on standard, prospectively determined definitions. If the invasive cardiac catheterization report was inconclusive, catheterization films were reviewed for a visual assessment of CAD. The decisions of the CEC will be used to perform the final statistical analyses.

The Primary Endpoint is the time to first event using the composite of the following major cardiovascular events:

All-cause mortality was used rather than cardiac mortality to eliminate the need for possibly difficult adjudication of causes of death, especially given the relatively low mortality expected.

Myocardial infarction (MI) is defined as an abnormal cardiac biomarker level above the institutional upper limit of normal (either troponin or CK-MB), and either ischemic discomfort lasting at least 10 minutes or ECG changes indicative of ischemia or infarction or new abnormal Q waves consistent with infarction. Additionally peri-procedural infarctions are defined as greater than three times the upper limit of normal for serum CK-MB following PCI and greater than five times the upper limit of normal following CABG.

Major complications from cardiovascular procedures and diagnostic testing which occur within 72 hours, including:

Stroke is defined as an acute focal neurological deficit of sudden onset, not reversible within 24 hours, or that resolves in $<24$ hours with clear evidence of a new stroke on cerebral imaging.

Bleeding is defined as major based on one or more of the following: Transfusion of $\geq 2$ units heterologous packed red blood cells or whole blood, decrease in hemoglobin level by $\geq 2.0 \mathrm{~g} / \mathrm{l}$, need for re-operation or invasive intervention (e.g. Evacuation of wound hematoma), or bleeding at a critical anatomic site (intracranial, intraspinal, intraocular, retroperitoneal, intra-articular, pericardial, or intramuscular with compartment syndrome)

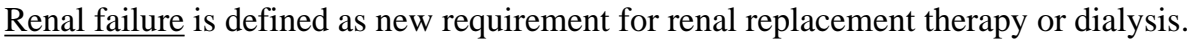

Anaphylaxis is defined as a severe contrast reaction requiring emergency respiratory and/or circulatory support.

Unstable angina hospitalization defined as an event in which the final diagnosis is myocardial ischemia and either of the following criteria are present: ischemic discomfort or equivalent symptoms requiring hospitalization within 48 hours of symptoms and lasting at least 10 minutes at rest, or ischemic discomfort or equivalent symptoms occurring in an accelerated pattern within 48 hours of hospitalization. In addition at least one of the following must be present: dynamic ST depression, ischemia on stress testing or significant epicardial coronary artery stenosis

\section{The Secondary Endpoints are}

- Death or MI or unstable angina hospitalization 
- Death or MI

- Major complications from cardiovascular procedures and testing (stroke, major bleeding, anaphylaxis, and renal failure requiring dialysis)

- Composite endpoint consisting of the primary endpoint or invasive catheterization without obstructive CAD (defined as all stenoses less than $50 \%$ in all major epicardial vessels including side branches $\geq 2 \mathrm{~mm}$ in diameter, on the first cath performed $<=60$ days after randomization.)

- Invasive catheterization without obstructive CAD defined as the site reported rate of all major epicardial vessels having less than $50 \%$ stenosis on the first cardiac catheterization procedure occurring within 60 days from randomization. Patients with structural causes for ischemia such as anomalous coronary arteries are excluded.

- Medical costs, resource use, and incremental cost effectiveness

- Health-related quality of life (QOL)

The Secondary Safety Endpoint is estimated cumulative radiation exposure as measured by cumulative radiation exposure in milliSieverts in each arm for tests performed for the diagnostic work-up during the 60 days using administered dose on CCTA, converted contrast agent dose for nuclear and administered radiation dose (kerma air product or dose length product) or fluoroscopy time for angiography. In addition, cumulative radiation exposure over the entire trial will be estimated by extrapolating the original data collection (average dose per test for each site) for exposures during follow-up.

Quality of Life assessments include the Seattle Angina Questionnaire (SAQ), the Duke Activity Status Index (DASI), the Rose Dyspnea Scale, the Medical Outcomes Study Short Form (SF-12), the Patient Health Questionnaire (PHQ), the EuroQoL-5D (EQ-5D), and the Stanford Presenteeism Scale.

Blood based Biomarkers and Image Repositories were created as enduring resources. Blood samples from consenting subjects were banked for clinical and advanced molecular biomarkers (DNA, RNA, metabolomics, proteomics). The Image Repository includes all CCTA, nuclear, echocardiographic and angiographic images and all stress ECGs.

\section{Statistical methods}

\section{Sample size and power calculations}

Based on the distribution of coronary disease expected in the study population (approximately 15\% obstructive disease, $40 \%$ non-obstructive disease, and $45 \%$ normal coronary arteries), and based on national claims data and published information from other databases, the event rate at 2.5 years (the approximate average length of follow-up) for the primary composite endpoint in subjects randomized to the functional testing strategy was projected to be approximately $9 \%$. $(4,8,13-15,18)$ 
The sample size of 10,000 patients was chosen to achieve (a) $\geq 90 \%$ power for detecting a $20 \%$ reduction in the primary endpoint if the 2.5 -year event rate in the functional testing arm is $8 \%$ or higher, (b) $80 \%$ power if the event rate is as low as $6 \%$, (c) adequate power for detecting a smaller reduction (16-17\%) if the event rate in the functional testing arm is 8$9 \%$ or higher, and (d) acceptable power for selected secondary endpoints. This number also provides $90 \%$ power for testing non-inferiority with a pre-specified margin of 1.10 (expressed as a hazard ratio of CCTA vs. functional testing) assuming that anatomic testing is only better than functional testing by $10 \%$. This study size allows for a $3 \%$ loss to followup. Under these various assumptions, this sample size will provide robust statistical power for assessing clinically relevant outcome differences between the two testing strategies.

\section{Primary statistical analysis}

All major treatment comparisons between the randomized groups will be performed according to the principle of "intention-to-treat"; that is, subjects will be analyzed (and endpoints attributed) according to the diagnostic testing strategy to which subjects were randomized, regardless of actual initial test or subsequent additional testing or postrandomization treatment and medical care. Statistical comparisons will be performed using 2 -sided significance tests. The primary statistical comparison will be a "time-to event" analysis using the Cox proportional hazards model and Kaplan-Meier survival estimates. To appropriately account for heterogeneity among the subjects, the Cox model will be adjusted for a pre-specified set of prognostically important baseline covariates including age, sex, CAD risk equivalent (diabetes, peripheral or cerebrovascular disease), and chest pain characteristics. The level of significance for the assessment of the primary endpoint will be $a=0.05$. If the study does not meet the superiority significance criterion, noninferiority of CCTA will be tested and established if the upper 95\% CI for the estimated hazard ratio falls below 1.10 .

\section{Diagnostic testing analyses}

Diagnostic test quality assurance analyses will include technical quality, which will be compared among the different testing modalities to characterize, describe, and assess any differences in overall quality among the various testing modalities, and the quality of test interpretations using both unweighted and weighted Kappa statistics. Pre-specified analyses of diagnostic testing accuracy include: diagnostic accuracy, prognostic performance and concordance between the site and core lab interpretations. Diagnostic accuracy will be assessed using conventional measures (sensitivity, specificity, and receiver operating characteristic [ROC] curves) in subjects undergoing cardiac catheterization. Corrected values of sensitivity, specificity, and the ROC curve will be estimated after adjusting for verification bias. $(31,32)$

\section{QOL analysis}

For each of the QOL measures examined in this study, data analysis will proceed in several stages. Initially, we will provide simple descriptive and comparative analyses by intentionto-treat. A nonparametric bootstrap will be used to estimate treatment differences with $95 \%$ confidence intervals (CI) and p-values. Since there is currently no consensus in the statistical literature about the best way to deal with the multiple comparisons problem arising from 
testing each individual scale at each time point separately, we propose two complementary approaches. First, we have pre-specified the angina frequency, and angina QOL scales from the SAQ and functional status using the Duke Activity Status Index as the primary QOL comparisons of interest and assign all other comparisons to a secondary (descriptive) status. Second, we will fit a mixed effect longitudinal proportional odds model(33) to model the time profile (fixed effect) using a restricted cubic spline function. Using the fitted model, we can estimate and compare QOL overall (global hypothesis of no difference anywhere) and the QOL average over follow-up (difference in the areas under the two QOL treatment curves), as well as make comparisons at selected points of interest, such as at end of study or at 6 months or 1 year. Missing data will be addressed using multiple imputation (missing at random assumption) and sensitivity analyses (cannot assume missing at random), as needed. No formal adjustment of $\mathrm{p}$ values for multiple comparisons will be made.

Economic analysis will consist of an empirical intention-to-treat cost comparison and costeffectiveness analysis. Confidence limits around the observed cost differences will be constructed using bootstrap methods. The cost-effectiveness analyses will estimate the incremental cost required to add an extra life year in the anatomic vs functional testing arm. In secondary analyses, we will incorporate utility weights to estimate the incremental cost per quality-adjusted life year gained with the CCTA anatomic strategy, relative to the functional testing strategy. These analyses will be conducted from a societal perspective and will use a lifetime time horizon so that the estimated incremental cost-effectiveness and cost-utility ratios can be compared with societal benchmarks.

\section{Organization, Funding and Human Studies (Appendix 1)}

PROMISE is funded by the National Heart, Lung, and Blood Institute (NHLBI). It is conducted under the supervision of an executive committee, which developed the protocol and oversees trial execution, the independent database, and analysis of the results and publication. NHLBI appointed an independent data and safety monitoring board to monitor patient safety and provide recommendations regarding terminating, continuing, or modifying the study protocol if concerns arise. A steering committee of investigators provides operational expertise and advises the executive committee. Finally, operational oversight of the study is performed through the Duke Clinical Research Institute, with Massachusetts General Hospital serving as the imaging and testing coordinating center. The Duke, Partners Healthcare and Tufts Institutional Review Boards approved the central activities; local or central IRBs approved the study at each site. Each patient was provided with oral and written information and signed a declaration of informed consent.

\section{Results}

PROMISE recently completed enrollment of the pre-specified 10,000 subjects in 3.2 years, ahead of the enrollment plan. Enrolling sites included 193 US and Canadian centers representing cardiology, radiology, primary care, urgent care and anesthesiology expertise. 


\section{Discussion}

The PROMISE trial enrolled a study cohort broadly reflective of the current population undergoing evaluation for stable symptoms suspicious for coronary artery disease which should provide a highly representative reflection of the current state of clinical diagnostic testing in North America, of subsequent care including additional testing, medical therapy and possible revascularization as well as cardiovascular events, costs of care, quality of life and radiation safety. Enrollment was completed expeditiously, ahead of the projected timeline, indicating that a site selection strategy involving multi-specialty community practices is both feasible and efficient.

Current ACC/AHA clinical practice guidelines on stable ischemic heart disease(3) provide two Class I diagnostic recommendations for patients able to exercise: one for stress ECG (Level of Evidence A), and the other for exercise nuclear scan or exercise echocardiography in patients with an uninterpretable rest ECG (Level of Evidence B). CCTA is a Class IIb recommendation (Level of Evidence B). For patients unable to exercise, the guidelines provide a Class 1 recommendation for pharmacological stress with MPI or echocardiography (Level of Evidence B) and a Class IIb recommendation for CCTA (Level of Evidence $\mathrm{C}$ ). These recommendations reflect the multitude of testing options available, the lack of high quality data and the paucity of guidance provided for choosing among available options in individual patients. Each of these factors contributes importantly to the rationale for the PROMISE study.

Recent investigations of populations undergoing functional testing for suspected CAD indicate a lower prevalence of CAD than in prior decades, as reflected in a much lower positivity rate(9) and few subsequent procedures or events.(8) These factors combine to result in high rates of angiographic findings of no obstructive coronary artery disease.(10, 34) Standards for test performance which were developed based on data from the 1990's(6) may substantially underestimate the false positive rate associated with contemporary referral patterns, favoring alternative modes of testing with higher sensitivity and specificity, such as CCTA.

PROMISE is the first large randomized trial of any form of noninvasive testing in cardiovascular disease, and among the first to use clinical events as its primary endpoint. As such PROMISE will test the outcomes research paradigm for cardiovascular imaging, a significant methodological question with broad policy implications.(23) The PROMISE trial is also breaking new ground in its pragmatic, clinical effectiveness design. By largely allowing sites to perform usual testing protocols and interpretation as well as site-directed, rather than protocol mandated, medical care following test results, PROMISE will yield much valuable information about current real world care in North America. However, it will not provide information regarding the efficacy of testing in expert hands or clinical outcomes following rigorously controlled subsequent care. As a trial of a diagnostic strategy, this study will address questions related to the optimal evaluation of symptomatic patients, rather than rigorously evaluating a diagnostic or therapeutic intervention. The clear requirement for site qualification prior to enrollment and maintenance of reasonable quality of test performance should ensure quality throughout the study. Site care teams are also 
expected to follow current guidelines for primary and secondary prevention during follow up.

\section{Summary and Significance}

PROMISE is the first large randomized trial comparing functional and anatomic noninvasive diagnostic strategies for the initial assessment of stable symptoms suspicious for possible $\mathrm{CAD}$, with the primary endpoint being superior health outcomes. It is expected to yield definitive and unique evidence regarding the benefits and risks of these alternative approaches.

\section{Abbreviations}

CAD coronary artery disease

CCTA coronary computed tomography angiogram

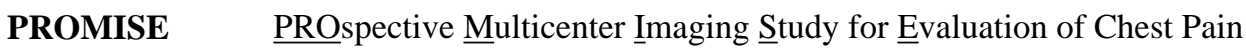

QOL quality of life

\section{References}

1. Go AS, Mozaffarian D, Roger VL, et al. Heart disease and stroke statistics--2013 update: a report from the American Heart Association. Circulation. 2013; 127(1):e6-e245. [PubMed: 23239837]

2. Selker HP, Zalenski RJ, Antman EM, et al. An evaluation of technologies for identifying acute cardiac ischemia in the emergency department: a report from a National Heart Attack Alert Program Working Group. Ann Emerg Med. 1997; 29(1):13-87. [PubMed: 8998086]

3. Fihn SD, Gardin JM, Abrams J, et al. 2012 ACCF/AHA/ACP/AATS/PCNA/SCAI/STS Guideline for the diagnosis and management of patients with stable ischemic heart disease: a report of the American College of Cardiology Foundation/American Heart Association Task Force on Practice Guidelines, and the American College of Physicians, American Association for Thoracic Surgery, Preventive Cardiovascular Nurses Association, Society for Cardiovascular Angiography and Interventions, and Society of Thoracic Surgeons. J Am Coll Cardiol. 2012; 60(24):e44-e164. [PubMed: 23182125]

4. Non-invasive cardiac imaging technologies for the diagnosis of coronary artery disease: a summary of evidence-based analyses. Ont Health Technol Assess Ser. 2010; 10(7):1-40.

5. Shaw LJ, Marwick TH, Zoghbi WA, et al. Why all the focus on cardiac imaging? JACC Cardiovasc Imaging. 2010; 3(7):789-94. [PubMed: 20633864]

6. Fleischmann KE, Hunink MG, Kuntz KM, et al. Exercise echocardiography or exercise SPECT imaging? A meta-analysis of diagnostic test performance. JAMA. 1998; 280(10):913-20. [PubMed: 9739977]

7. Mowatt G, Vale L, Brazzelli M, et al. Systematic review of the effectiveness and cost-effectiveness, and economic evaluation, of myocardial perfusion scintigraphy for the diagnosis and management of angina and myocardial infarction. Health Technol Assess. 2004; 8(30):iii-iv. 1-207. [PubMed: 15248938]

8. Mudrick DW, Cowper PA, Shah BR, et al. Downstream procedures and outcomes after stress testing for chest pain without known coronary artery disease in the United States. Am Heart J. 2012; 163(3):454-61. [PubMed: 22424017]

9. Rozanski A, Gransar H, Hayes SW, et al. Temporal trends in the frequency of inducible myocardial ischemia during cardiac stress testing: 1991 to 2009. J Am Coll Cardiol. 2013; 61(10):1054-65. [PubMed: 23473411]

10. Patel MR, Peterson ED, Dai D, et al. Low diagnostic yield of elective coronary angiography. N Engl J Med. 2010; 362(10):886-95. [PubMed: 20220183] 
11. Iglehart JK. The new era of medical imaging--progress and pitfalls. N Engl J Med. 2006; 354(26): 2822-8. [PubMed: 16807422]

12. Shaw L, Narula J. From adequate evidence to optimal evidence. JACC Cardiovasc Imaging. 2012; 5(12):1292-3. [PubMed: 23236983]

13. Budoff MJ, Dowe D, Jollis JG, et al. Diagnostic performance of 64-multidetector row coronary computed tomographic angiography for evaluation of coronary artery stenosis in individuals without known coronary artery disease: results from the prospective multicenter ACCURACY (Assessment by Coronary Computed Tomographic Angiography of Individuals Undergoing Invasive Coronary Angiography) trial. J Am Coll Cardiol. 2008; 52:1724-32. [PubMed: 19007693]

14. Meijboom WB, Meijs MFL, Schuijf JD, et al. Diagnostic accuracy of 64-slice computed tomography coronary angiography: a prospective, multicenter, multivendor study. J Am Coll Cardiol. 2008; 52:2135-44. [PubMed: 19095130]

15. Miller JM, Rochitte CE, Dewey M, et al. Diagnostic performance of coronary angiography by 64row CT. N Engl J Med. 2008; 359(22):2324-36. [PubMed: 19038879]

16. Schuetz GM, Zacharopoulou NM, Schlattmann P, et al. Meta-analysis: noninvasive coronary angiography using computed tomography versus magnetic resonance imaging. Ann Intern Med. 2010:167-77. [PubMed: 20124233]

17. Hadamitzky M, Distler R, Meyer T, et al. Prognostic value of coronary computed tomographic angiography in comparison with calcium scoring and clinical risk scores. Circ Cardiovasc Imaging. 2011:16-23. [PubMed: 20884832]

18. Bamberg F, Sommer WH, Hoffmann V, et al. Meta-analysis and systematic review of the longterm predictive value of assessment of coronary atherosclerosis by contrast-enhanced coronary computed tomography angiography. J Am Coll Cardiol. 2011; 57:2426-36. [PubMed: 21658564]

19. Hulten EA, Carbonaro S, Petrillo SP, et al. Prognostic value of cardiac computed tomography angiography: a systematic review and meta-analysis. J Am Coll Cardiol. 2011:1237-47. [PubMed: 21145688]

20. Shreibati JB, Baker LC, Hlatky MA. Association of coronary CT angiography or stress testing with subsequent utilization and spending among Medicare beneficiaries. JAMA. 2011; 306(19):212836. [PubMed: 22089720]

21. Tonino PA, De Bruyne B, Pijls NH, et al. Fractional flow reserve versus angiography for guiding percutaneous coronary intervention. N Engl J Med. 2009; 360(3):213-24. [PubMed: 19144937]

22. Lauer MS. CT angiography: first things first. Circ Cardiovasc Imaging. 2009; 2(1):1-3. [PubMed: 19808556]

23. Douglas PS, Taylor A, Bild D, et al. Outcomes research in cardiovascular imaging: report of a workshop sponsored by the National Heart, Lung, and Blood Institute. JACC Cardiovasc Imaging. 2009; 2(7):897-907. [PubMed: 19608141]

24. Hlatky MA, Douglas PS, Cook NL, et al. Future directions for cardiovascular disease comparative effectiveness research: report of a workshop sponsored by the National Heart, Lung, and Blood Institute. J Am Coll Cardiol. 2012; 60(7):569-80. [PubMed: 22796257]

25. Chalkidou K, Tunis S, Whicher D, et al. The role for pragmatic randomized controlled trials (pRCTs) in comparative effectiveness research. Clin Trials. 2012; 9(4):436-46. [PubMed: 22752634]

26. Beller GA, Bonow RO, Fuster V. ACCF 2008 Recommendations for Training in Adult Cardiovascular Medicine Core Cardiology Training (COCATS 3) (revision of the 2002 COCATS Training Statement). J Am Coll Cardiol. 2008; 51(3):335-8. [PubMed: 18206749]

27. Pellikka PA, Nagueh SF, Elhendy AA, et al. American Society of Echocardiography recommendations for performance, interpretation, and application of stress echocardiography. $\mathrm{J}$ Am Soc Echocardiogr. 2007; 20(9):1021-41. [PubMed: 17765820]

28. Nichols KJ, Bacharach SL, Bergmann SR, et al. Instrumentation quality assurance and performance. Journal of Nuclear Cardiology. 2007; 14(6):e61-e76.

29. Raff GL, Abidov A, Achenbach S, et al. SCCT guidelines for the interpretation and reporting of coronary computed tomographic angiography. J Cardiovasc Comput Tomogr. 2009; 3(2):122-36. [PubMed: 19272853] 
30. Abbara S, Arbab-Zadeh A, Callister TQ, et al. SCCT guidelines for performance of coronary computed tomographic angiography: a report of the Society of Cardiovascular Computed Tomography Guidelines Committee. J Cardiovasc Comput Tomogr. 2009; 3(3):190-204. [PubMed: 19409872]

31. Kosinski AS, Barnhart HX. A global sensitivity analysis of performance of a medical diagnostic test when verification bias is present. Stat Med. 2003; 22(17):2711-21. [PubMed: 12939781]

32. Alonzo TA, Pepe MS. Assessing accuracy of a continuous screening test in the presence of verification bias. Journal of the Royal Statistical Society: Series C (Applied Statistics). 2005; 54(1):173-90.

33. Hedeker D, Gibbons RD. A random-effects ordinal regression model for multilevel analysis. Biometrics. 1994; 50(4):933-44. [PubMed: 7787006]

34. Thomas GS, Voros S, McPherson JA, et al. A blood-based gene expression test for obstructive coronary artery disease tested in symptomatic nondiabetic patients referred for myocardial perfusion imaging the COMPASS study. Circ Cardiovasc Genet. 2013; 6(2):154-62. [PubMed: 23418288]

\section{Appendix 1. Trial Organization}

\section{Executive Committee}

Pamela S. Douglas, MD

Lawton Cooper, MD

Udo Hoffmann, MD, MPH

Kerry Lee, $\mathrm{PhD}$

Daniel Mark, MD, MPH

\section{Data and Safety Monitoring Board}

Robert Bonow, MD

Garnet Anderson, $\mathrm{PhD}$

Alain Bertoni, MD, MPH

J. Jeffrey Carr, MD

James K. Min, MD

Michael Proschan, PhD

John A. Spertus, MD, MPH

Connie M. Ulrich, $\mathrm{PhD}, \mathrm{RN}$

\section{Operations Steering Committee (in addition to Executive Committee)}

Hussein R. Al-Khalidi, PhD

Denise Bonds, MD 
Nakela Cook, MD

Rowena J. Dolor, MD, MHS

Alan Go, MD

Tina Harding

Sarah Hayden

Andrzej Kosinski, $\mathrm{PhD}$

Mitchell W. Krucoff, MD

Eric Leifer, $\mathrm{PhD}$

Daniel W. Mudrick, MD, MPH

Manesh R. Patel, MD

Michael Picard, MD

Geoffrey Rubin, MD

Kristen Salvaggio

Ricky Schneider, MD

Alexandra Shen, MD

Jean Claude Tardiff, MD

James E. Udelson, MD

John Vavalle, MD

Eric J. Velazquez, MD

\section{Diagnostic Testing Coordinating Center}

Udo Hoffmann, MD, MPH

Charles Apgar, MBA

Kristen Salvaggio

James E. Udelson, MD

\section{Core Laboratories}

CCTA: Udo Hoffmann, MD, MPH (PI) 
Stephan Achenbach, MD (overreads)

\section{Erin Corsini}

Brian B. Ghoshhajra, MD MBA (sites, radiation)

Quynh Truong MD MPH (online reader certification)

Nuclear imaging: James E. Udelson, MD

Stress echocardiography; Michael H. Picard, MD

Stress ECG: Mitchell W. Krucoff, MD 


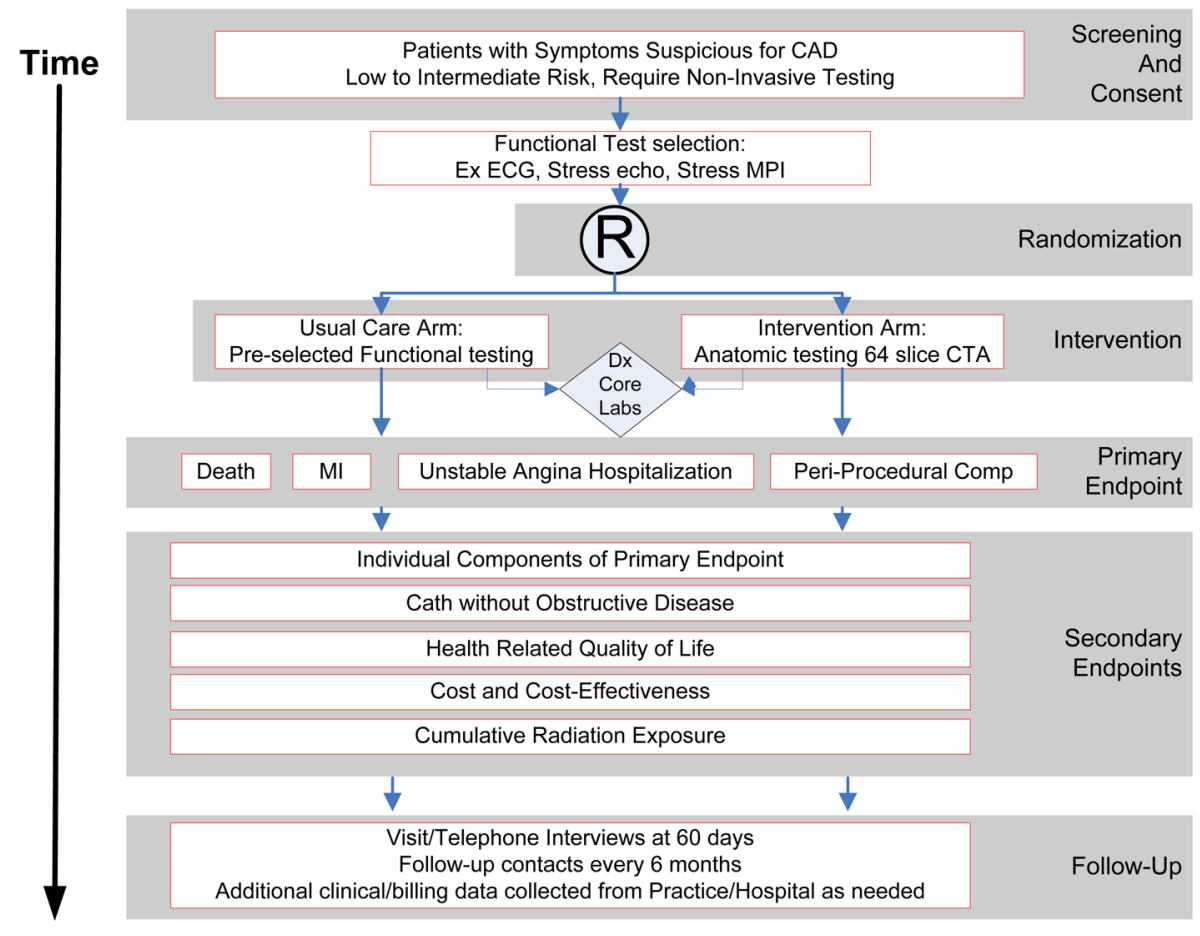

Figure 1. Overall Study Design

$\mathrm{CAD}=$ Coronary artery disease $; \mathrm{Ex}$ ECG $=$ Exercise ECG; Stress echo $=$ Stress echocardiography; MPI= Myocardial perfusion imaging; $\mathrm{Dx}=$ Diagnostic. 
Table 1

\section{Inclusion and Exclusion Criteria}

\begin{tabular}{|c|c|}
\hline Inclusion Criteria & Exclusion Criteria \\
\hline 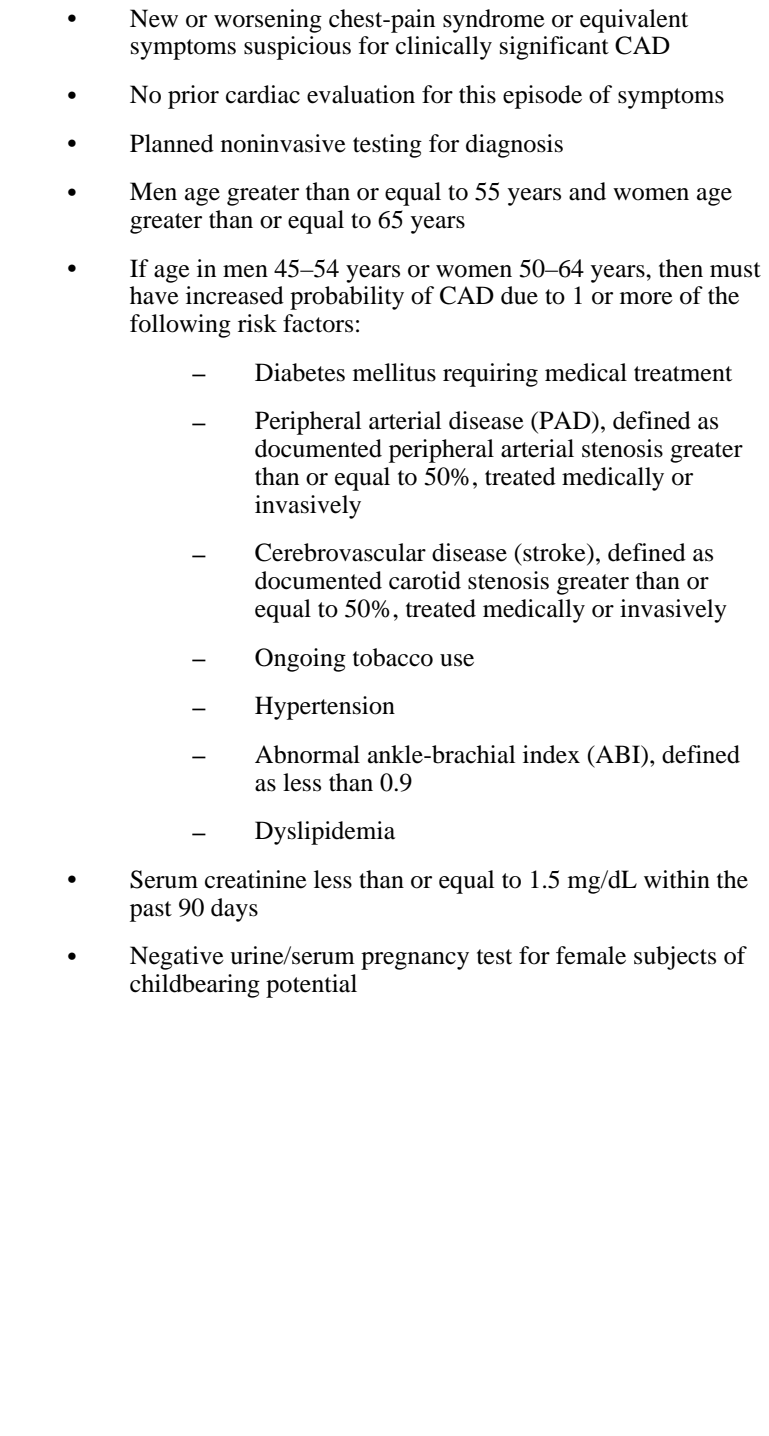 & 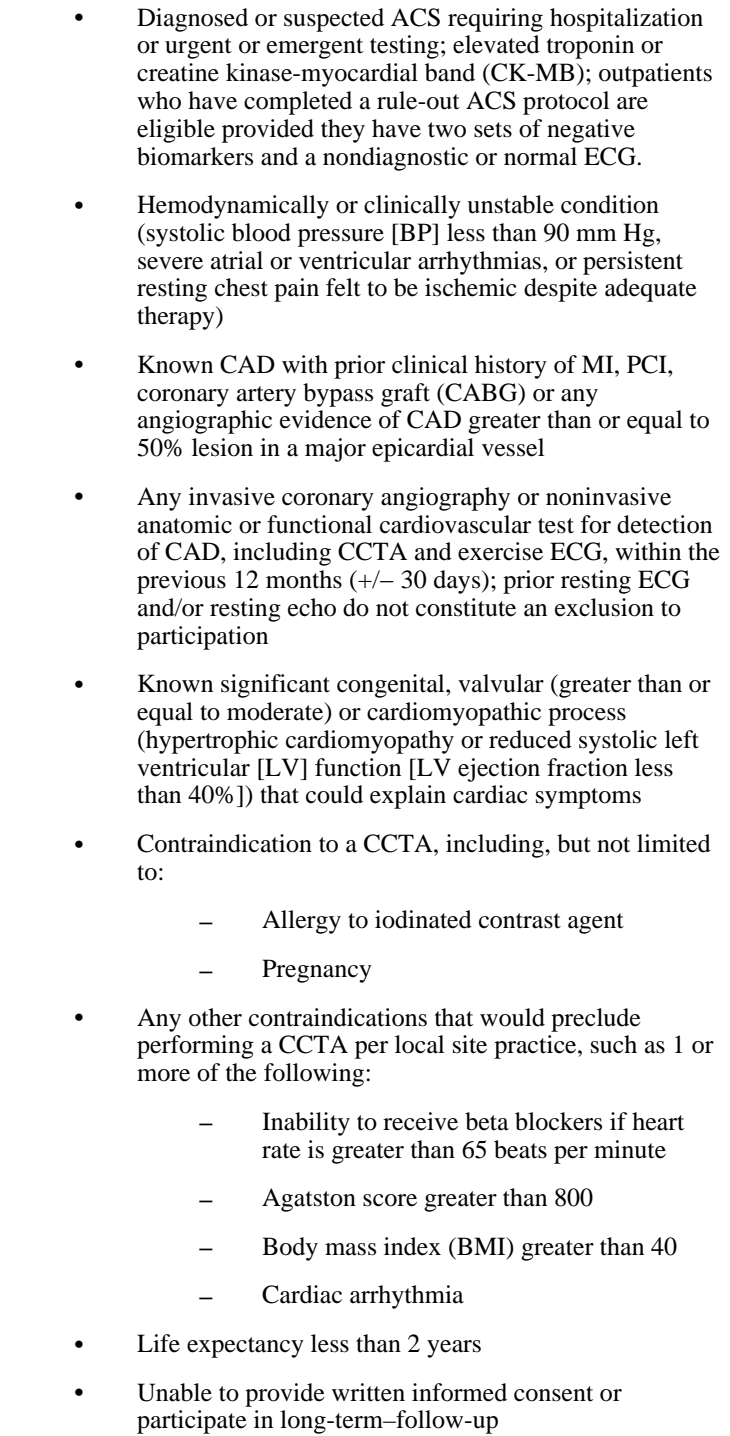 \\
\hline
\end{tabular}

